\title{
23. Functional Histopathology
}

\begin{tabular}{|l|l|}
\hline C. & Cordon-Cardo \\
\hline H.F. & Oettgen \\
\hline M.R. & Melamed \\
\hline
\end{tabular}

Departments of Pathology and Clinical Immunology, Memorial Sloan-Kettering Cancer Center, New York, N.Y., USA

C. Cordon-Cardo, Department of Pathology, Memorial Sloan-Kettering Cancer Center, 1275 York Avenue, New York, NY 10021 (USA)

Presented June 1986

Cancers of unknown primary site (CUPS) present major diagnostic and therapeutic problems. The incidence of metastatic malignant diseases of undetermined origin ranges between 0.5 and $7.0 \%$, depending upon the scope and duration of the diagnostic investigation [1]. CUPS account for as many as $10-15 \%$ of the referred patients with solid tumors in major cancer centers [2]. Despite extensive clinical investigation, the primary cancer site in a substantial number of these patients is unlikely to be found; in approximately 15-30\% of patients, a primary cancer site is not identified even at autopsy [3, 4].

Conventional histochemistry (i.e. special stains for tissue or cellular constituents based on dye binding or enzymatic reactions) has been used for many years and is of great value in identifying microorganisms, in studying the pathogenesis of certain disease processes and in the classification of some tumors. However, the chemical reactions involved do not generally distinguish among the many different compounds within a class of substances, and they are of limited value in tumor classification.

The recent introduction of immunocyto-chemical methods has provided a much more powerful tool for the identification of tissue and cellular constituents, and uses the high specificity and sensitivity of antibodies as probes for such tissue antigens. With this technique it has become apparent that certain cell constituents can now be readily detected and reflect cell type, differentiation or functional status among other properties.

It is becoming increasingly evident that differentiation antigens and tumor-associated antigens, as well as protein products encoded by oncogenes, play some role in the development of human cancer [5]. Because they reflect the origin and function of the tumor, monoclonal antibodies to these antigens are potentially of value to the pathologist to characterize and subclassify undifferentiated neoplasms and tumors with similar morphology but of different origin or clinical behavior. While it is possible that a single highly specific antibody will

178

Cordon-Cardo/Oettgen/Melamed

recognize a particular tumor, most cancers probably will require a battery of several antibodies for their identification and sub-classification. 
The value of immunocytochemistry in the histopathologic diagnosis of malignant tumors has been recently evaluated by different groups in a series of anaplastic and un-differentiated cancers, including cases of CUPS. Correlation between the initial histo-logic opinion and immunohistologic diagnosis resulted in the reclassification of these neoplasms as follows: $22 \%$ carcinoma cases, 67\% lymphoma cases, and $11 \%$ unclassified tumor lesions [6]. In another report, dealing with immunohistologic analysis of large cell undifferentiated tumors, the diagnoses were altered in $60 \%$ of the cases [7]. Furthermore, review of clinical details has shown that clinical management in several instances was influenced by establishing the correct diagnosis $[8,9]$.

There are several approaches to the care of patients with CUPS. One is to utilize chemotherapeutic regimens with agents active against the most likely primary cancer site, as well as those sites most responsive to chemotherapy. Adenocarcinomas are the commonest neoplasms identified, ranging between 24 and $48 \%$ of all cases [1]. Of those patients with CUPS in whom a primary cancer site is ultimately determined, cancer of the pancreas, lung and colon predominate. A smaller percentage have gastric cancer; less than $3 \%$ have more responsive tumors such as breast and ovarian cancers [1]. Malignant melanomas and sarcomas represent 5-7\% of the total number of CUPS with microscopically confirmed diagnoses $[1,10]$. Thus, although chemotherapy is a theoretically attractive concept, the cancers most likely to present as CUPS are also those tumors

highly refractory to conventional antineo-plastic agents.

Nonetheless, the role of the pathologist in providing a better and more accurate classification of neoplasms is an important one for the future. To such increased accuracy of diagnosis must be added the observation that, today, pathologists can identify a wide variety of functional and differentiation parameters such as receptors for growth factors and hormones. These observations, in time, should also assist in the better planning of appropriate therapeutic regimens.

References

Altman E, Cadman E: Cancer 1986;57:120-124.

Holmes FF, Fouts TL: Cancer 1970;26:816-820.

Stewart JF, Tattersall MHN: Br Med J 1979;i: 1530-1533.

Karsell PR, Sheedy MF, O’Connell MJ, et al: J Am Med Ass 1982;248:340-343.

Slamon DJ, deKernion JB, Verma IM, et al: Science 1984;224:256-262.

Gatter KC, Alcock C, Heryet A, Mason DY: Lancet 1985;i: 1302-1305.

Lauder I, Holland D, Mason DY, et al: Histopa-thology 1984;8:259-272.

Borowitz MJ, Stevanovic G, Gottfried M, et al.: HumPathol 1984;15:928-934.

Gatter KC, Alcok C, Heryet A, et al: Am J Clin Pathol 1984;82:33-43.

10 Cochran AJ, Wen DR, Herschman HR: Int J Cancer 1984;34:159-163. 